\title{
The Role of Kappa- and Mu-Opioid Receptors in Pruritus
}

Brian S. Kim, MD, MTR'1, Thomas Sciascia, MD², Gil Yosipovitch, MD³

'Washington University, St. Louis, MO, USA; ${ }^{2}$ Trevi Therapeutics, New Haven, CT, USA; ${ }^{3}$ University of Miami, Miami, FL, USA

\section{Introduction}

Background

Itch perception is transmitted from sensory neurons innervating the skin to the spinal cord; from there, spina projection neurons relay signals cord
sensation is perceived (Figure 1) A multitude of itch-inducing stimuli or pruritogens can
trigger itch, including neurotransmitters, neuropeptides, proteases, and cytokines' ${ }^{2}$; however, path
suppress itch remain poorly understood

Chronic pruritus, defined as itch persisting for 26 weeks, may arise from a range of dermatologic, systemic, neuropathic, and psy
challenging to treat

Although opioid receptors are typically associated with their role in pain signaling, recent studies have shed light kappa-opioid receptors (KOPs) and mu-opioid receptors (MORs), respectively, in suppressing and eliciting itch both in the periphery and more centrally

Objective

To summarize recent work supporting the role of KORs and MORs
treatment of itch

\section{Methods}

Aliterature search of the PubMed database was
conducted to identify English-language publications examining the role of opioid receptors in pruritus in the past decade (select references cited within identified

Search terms included "opioid receptor", "kappa".

Findings from relevant publications were summarized as
a narrative review

\section{Results} Itch Signaling Pathway and the Role of Opioid

Kappa- and mu-opioid receptors have been identified throughout the itch signaling pathway, from skin, to
spinal cord, to central nervous system (CNS; Figure 1), 1,1,2
Results (continued)

Figure 1. Presence of MORs and KORs Throughout the Itch Signaling Pathway

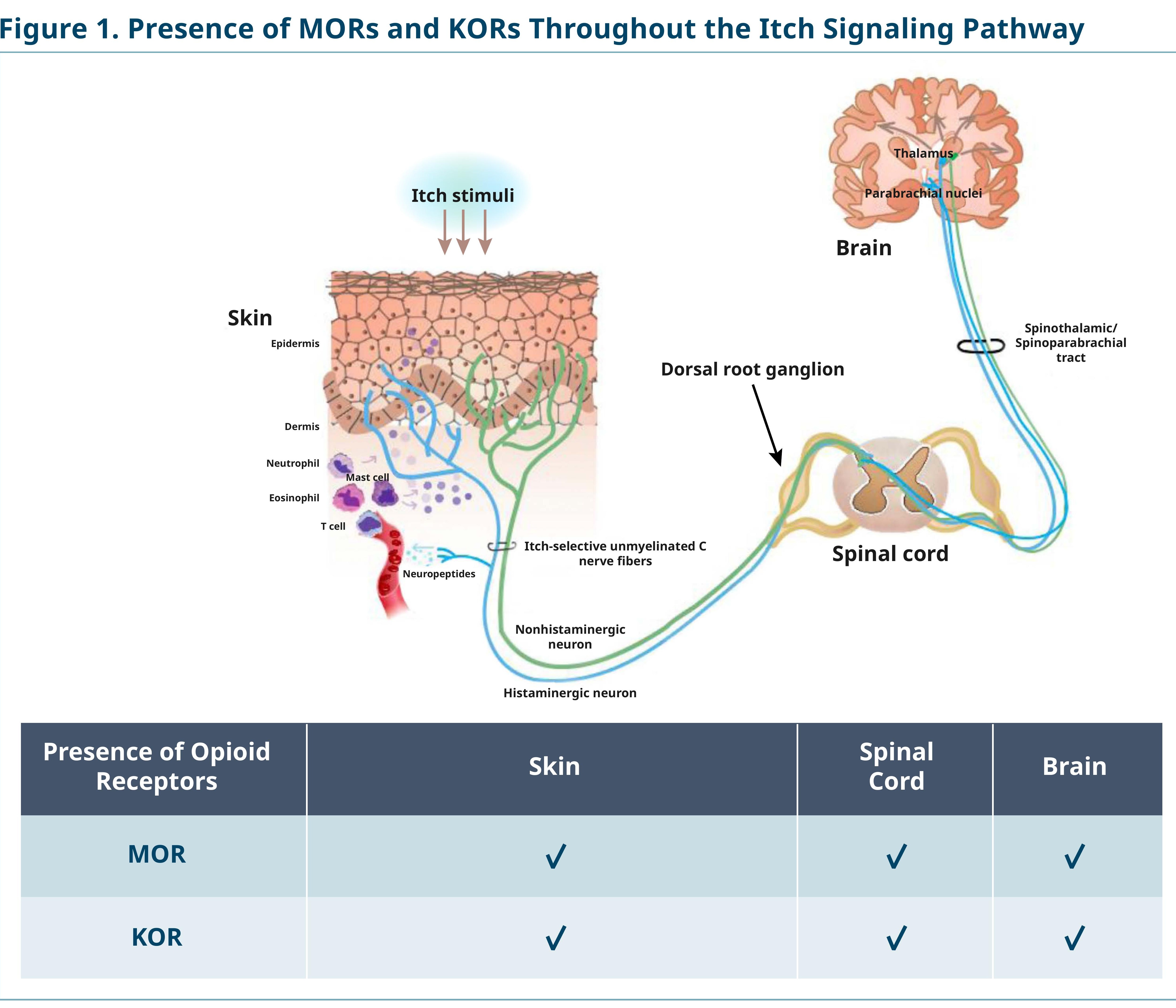

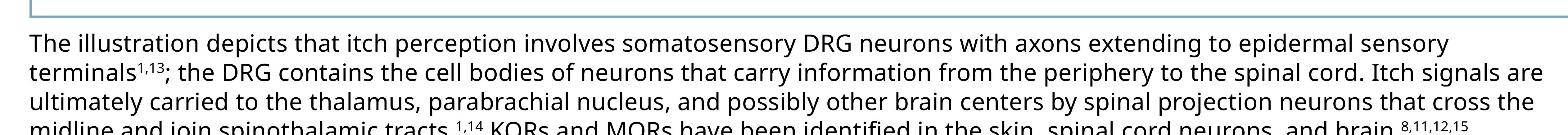

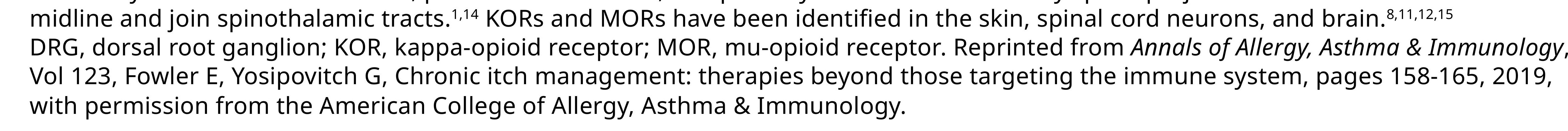
- Following binding of an opioid to an opioid receptor, a cascade of intracellular changes
occurs, resulting in reduced cellular excitability (Figure 2A $)^{16}$ Although activation of both KORs and MORs results in analgesia, other effects are
distinct (Figures $2 \mathrm{~B}$ and $2 \mathrm{C}$ ) In particular, whereas activation of $\mathrm{KORs}$ results in attenuation of itch7 in a variety of
contexts, activation of MORs is associated with increased itch ${ }^{17}$ (Figures $2 \mathrm{~B}$ and $2 \mathrm{C}$ ) In addition, there are reports of KOP agonism resulting in suppression of inflammation - Although the exact mechanisms are not established, in a preclinical model of atopic dermatitis, the dual KOR agonist/MOR antagonist nalbuphine decreased expression of the pruritogenic cytokine interleukin (II)
expression of the anti-inflammatory cytokine IL-11 $11^{18}$ In contrast to MOR activation, neither MOR blockade nor KOR activation are
associated with addiction," which has important therapeutic implications given

Imbalances of activity across the KOR and MOR systems in the skin or CNS are
associated with severe chronic pruritus and are an active area of research for associated with sever
novel treatments

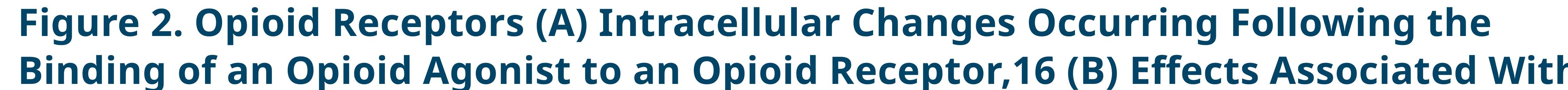
MOR Activation, (C) Effects Associated With KoR Activation

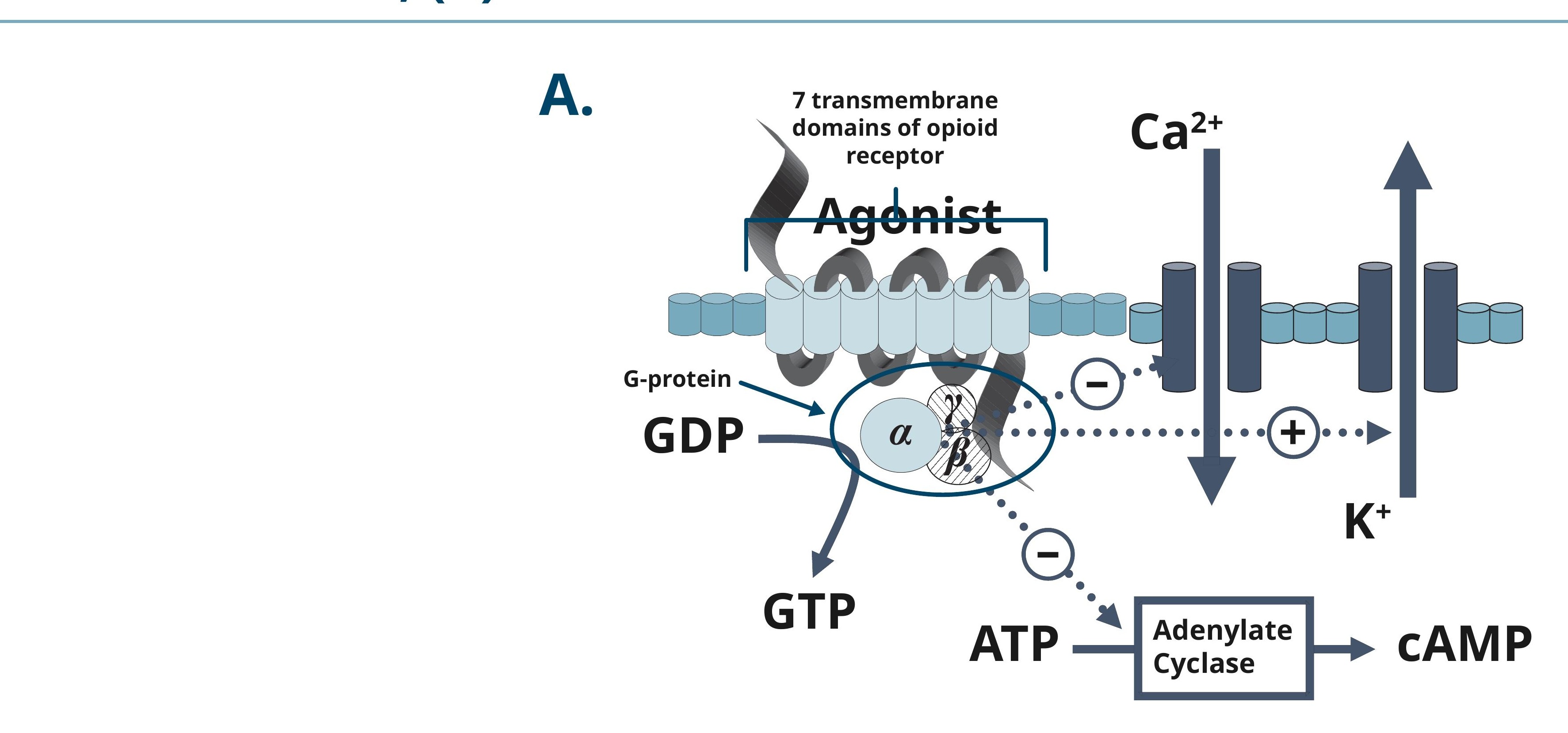

B.
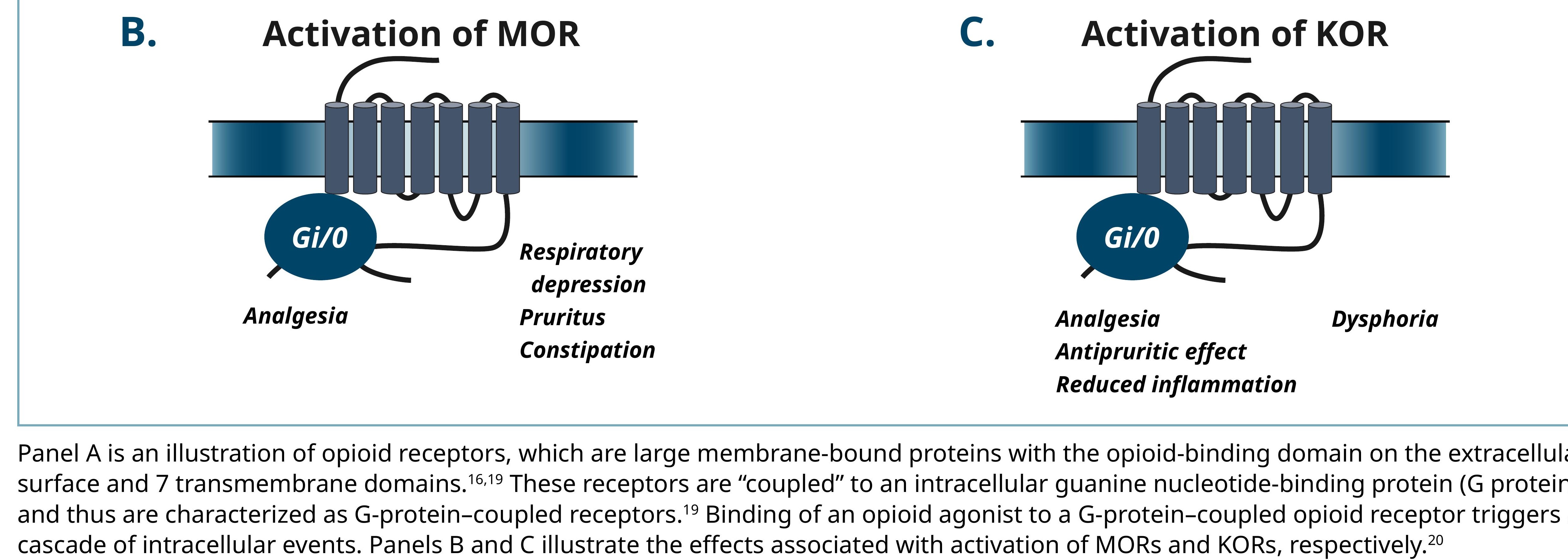

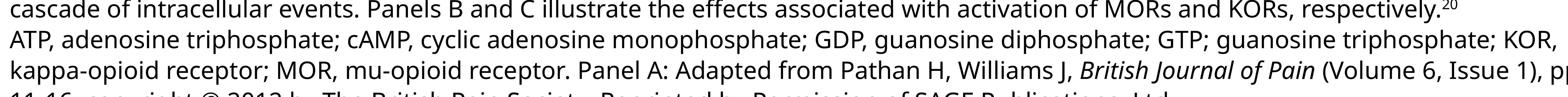

Preclinical Models Elucidate the Effects of KOR Activation

Kappa-opioid receptors are expressed on 2 different populations of dorsal root ganglion

- Trons associated with hair follicles in the epidermis The endogenous KOR agonist dynorphin reduces neuronal excitability
Dynorphin is produced by inhibitory interneurons that modulate the neurons
that respond to itch stimuli $1^{21}$

KORs and MORs as Therapeutic Targets

Agents that activate KORs have been shown to act within the peripheral nervous system

Attenuation of itch has been demonstrated by KOR agonists, including the
endogenous ligand dynorphin and drugs like nalfurafine and difelikefalin - The association of MOR activation with increased itching" supports blockade of MORs as another rational approach to inhibit itch

- Likewise, the MOR antagonist naltrexone is employed as an antipruritic agent

Both KOR and MOR pathways are targeted with use of dual KOR agonist/MOR
antagonists such as butorphanol and nalbuphine

Opioid agents targeting KORs and MORS (Figure 3) have demonstrated efficacy in a
variety of chronic pruritic conditions, including uremic pruritus and prurigo nodularis 3238

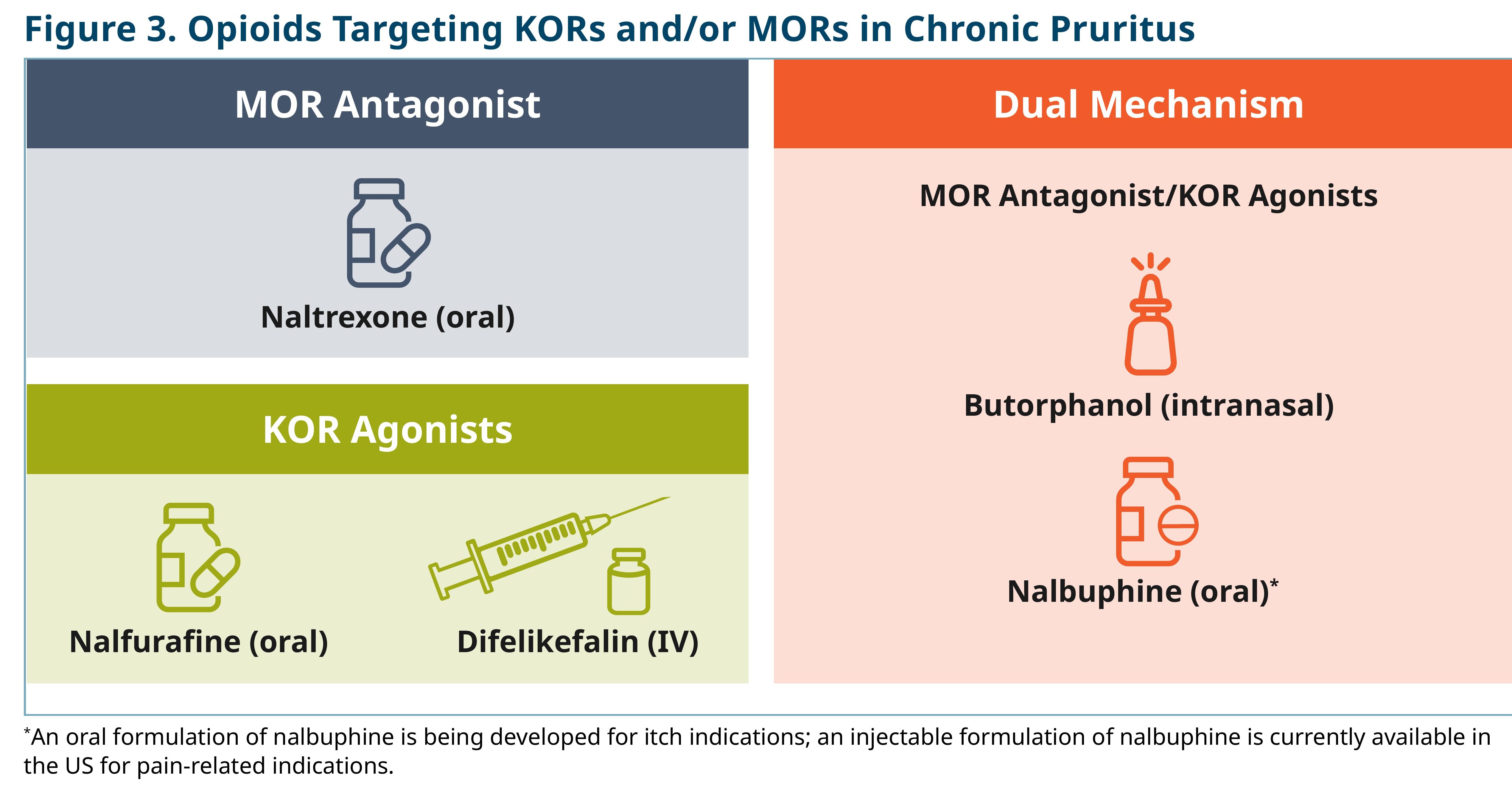

\section{Conclusions}

Kappa- and mu-opioid receptors have emerged as important therapeutic targets in itch Notwithstanding these advances, the precise mechanisms by which KOR agonists and/
or MOR antagonists can be employed therapeutically remains an exciting area worthy

\section{Disclosures}

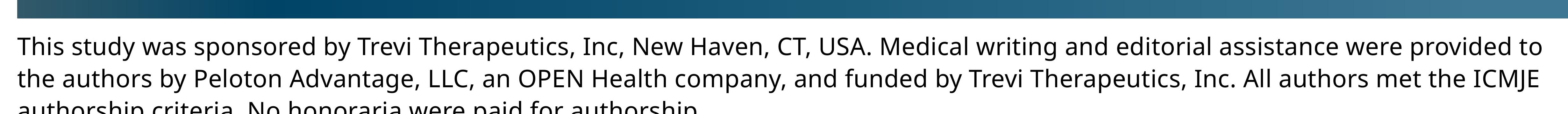

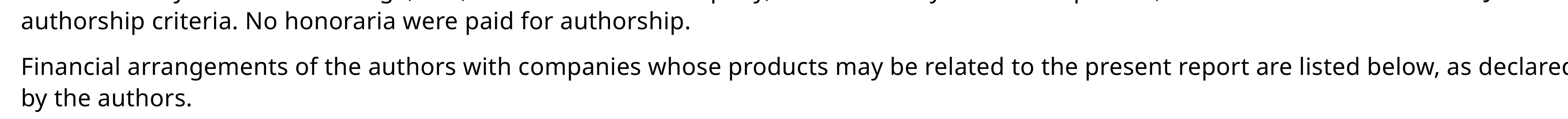

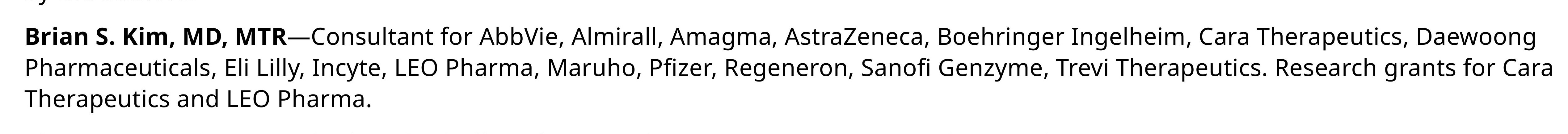

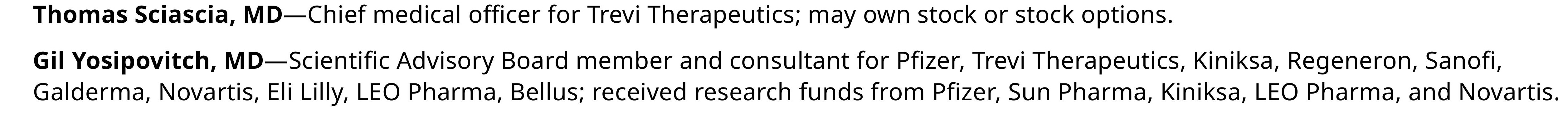

References

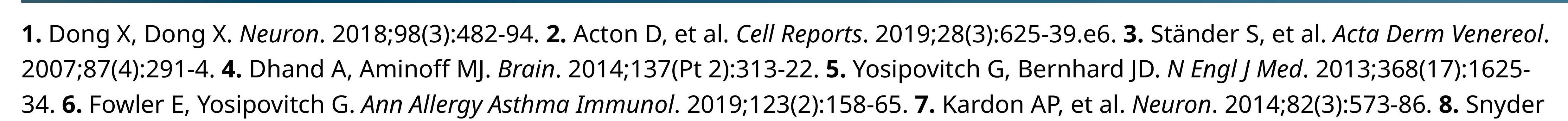

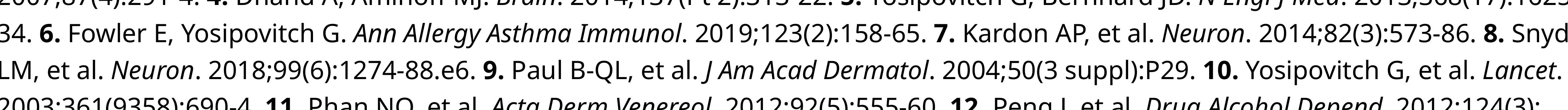

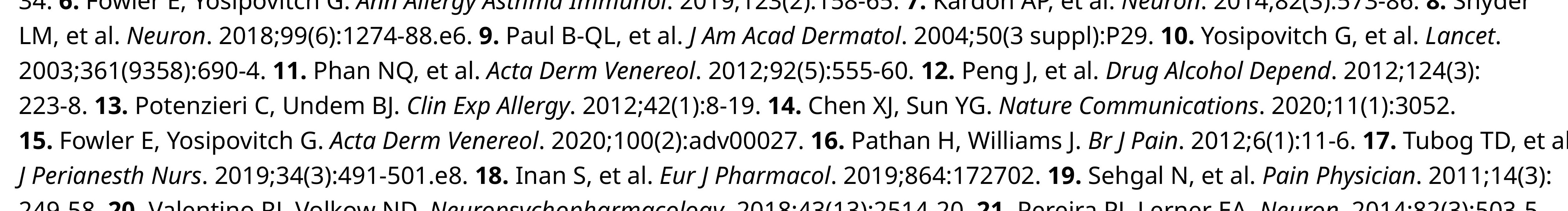

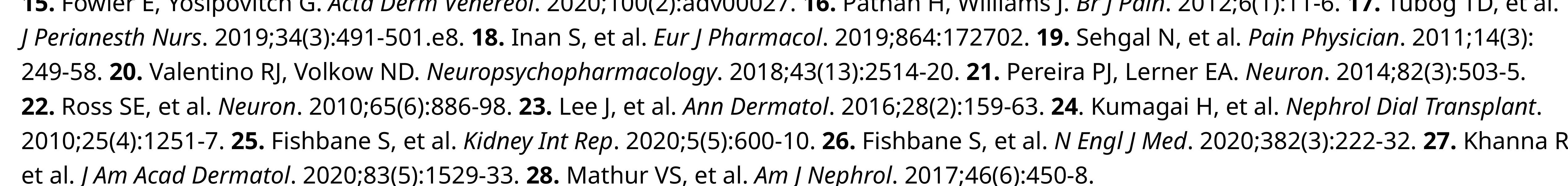

